\title{
Color design of the internal environment of factory workshop
}

\author{
Wei Chen", and Jun Fan \\ Jiangsu Open University, Nanjing, China
}

Keywords: color, workshop, environment.

\begin{abstract}
In view of the present situation that the factory workshop is in a grey environment world, to accelerate the fatigue of operators and affect the productivity of labor, this paper puts forward how to design the color of production environment from the angle of the human physiology and psychology. And points out that working under a good environment color can improve productivity and reduce labor accidents.
\end{abstract}

\section{Introduction}

At present, in many factories such as machinery and metallurgy in China, the interior of factories is mostly based on walls coated with white mortar. This kind of wall is not only easy to stick dust and pollution, but also covered by many production equipment in the factory building. The production equipment (such as various pipelines, bridge cranes, etc.) is mostly grey, and the products produced are often painted in grey or metallic primary colors. In this way, the whole workshop becomes a gray world[1]. Working in this environment gives people a feeling of crowding, depression and insecurity, which greatly depresses people's mood. People will soon be tired, to affect the processing accuracy and production efficiency. With the progress of the times and the development of science and technology, people's living standards have been greatly improved. It is recognized that besides beautifying the living environment, we should also pay attention to beautifying the production environment of the workplace so as to keep the operators in a vigorous mental state and reduce fatigue. Therefore, designers put forward higher requirements for the existing environment in the workshop. Creating a work environment with high labor efficiency and pleasant mood has become the requirement of the times[2].

Research shows that good environmental color mainly achieves the purpose of improving productivity and reducing labor accidents by improving human visual conditions (physiological factors) and human visual environment (psychological factors)[3]. Therefore, the color design of factory workshop interior environment is a very important issue, which must be paid enough attention to.

\footnotetext{
* Corresponding author: chenwei@jstvu.edu.cn
} 


\section{The physiological and psychological functions of colors on human beings}

Color is an indispensable visual feeling in people's life. In a sense, the world was colorless, but people feel that they live in a colorful world. Anyone with normal visual ability can see different combinations of colors as soon as he opens his eyes.

Color is the physiological and psychological function of human beings through human visual perception, is the subjective reflection of the objective material world to human beings. Color stimulates human to cause certain physiological changes accompanied by a certain psychological effect. Similarly, color causes psychological activities, but also produces certain physiological changes. That people see warm tones expands their pupils and accelerates their pulsation. Especially, yellow, orange, red and other exciting colors can give human vitality and excitement. When human see cold tones, they feel cool and cold psychologically, and their pulse is stable and their mood is stable. Psychologist experiments have proved that harmonious and beautiful colors can make human vigorous and spiritual pleasure. Too stimulating color can make human secrete a harmful substance of health and mood, to make them nervous disorders, emotional fluctuations, healthy damage.

The positive effect of color on human physiology and psychology can create a comfortable color environment for the workshop interior.

\section{The relationship between light and color in workshop}

Light and color have an inseparable close relationship. Without light, there is no sense of color. With light, people can see the shape and color of objects, to gain understanding of the objective world. If we are in a place where there is no light, no color can be recognized. Without light, there would be no visual activity, and of course there would be no sense of color. Therefore, light is the cause of color being caused, and color is the result of light being sensed. In this sense, color of producing the relationship among light, eye and brain is called color. To see color must have light, that is, color is the visual response of light.

There are natural light source (sunlight) and artificial light source (lighting lamps). In factory workshops, only natural light is not feasible because of the restriction of the structure of factory buildings, artificial light sources are also used. This kind of light environment composed of artificial light sources is called lighting.

Experiments show[4] that visual organs have different adaptability to different illumination (illumination is quantitative indicator of lighting design, indicating the intensity of light on the illuminated surface) in the process of light and color sensation, so their requirements for brightness are also different. Therefore, in order to lighten the burden of visual organs and reduce or eliminate visual fatigue, the illumination of the operating environment should be consistent. Experience shows that when people's vision changes from dark to light or from light to dark, there is a process of adaptation. In this process, it will consume a part of energy, to distract human attention and produce fatigue. So that, the indoor illumination should be uniform and consistent, and must be in harmony with the brightness of the wall color, production equipment color and other object color, there is no strong contrast, no sharp changes in light and shade.

Under the conditions of sunlight and lighting, the environmental color of factory buildings will change. Because the spectrum composition of artificial light source is different from that of natural light source, there are also differences in the display effect of colors. Light color has three different effects on color change, enlargement and reduction. Where the illumination is high, the color value is also high, to cause the color purity increase. Where the illumination is low, the opposite is true. Therefore, in the light, blue, 
blue-green, green series of colors make human feel bright. Red, orange and yellow series of colors make human feel gloom.

There are three types of artificial light sources: general lighting, local lighting and mixed lighting. Source of general lighting is distributed in large space, the lighting is more uniform, which is conducive to the coordination of color atmosphere in the whole production environment. It is suitable for people's visual requirements, but not very economical. Local lighting is concentrated light which can ensure that the local working point has sufficient brightness, but the light is not uniform, and easy to appear dazzling glare. When the line of sight is transferred from the working face to other places, human eyes are easy to fatigue. Mixed lighting is a combination of general lighting and local lighting. This kind of lighting is beneficial to the harmony of environmental color and the adaptability of human eyes. It is a relatively economical and reasonable lighting form.

In production, the poor treatment of environmental color in the workshop will also accelerate people's fatigue. In the spectrum, purple and blue are the most likely to cause eye fatigue, followed by red. In order to eliminate the fatigue caused by color, it is necessary to look at the complementary color to restore fatigue when human see a certain color for a long time. The brighter the color, the more tired the human vision is, and quickly lose the sensitivity to color. Ultimately, it can lead to the possibility of accidents. It is generally required that environmental color should be medium brightness.

Therefore, in the production environment inside the workshop, light and color are the material basis for creating a lively and comfortable working environment. If we deal with the relationship between light and color in the workplace, we can improve labor productivity, improve product quality, reduce waste products, reduce accidents at work and protect workers' health.

\section{Choice of interior color in workshop}

In fact, color is a physical phenomenon related to human psychological and physiological phenomena. It is one of the important means to express the environmental function. Whether the color inside the workshop is suitable or not is directly related to the improvement of productivity, the safety and physical and mental health of operators.

From the use function, workshop interior color can be divided into focus color, production equipment color and surrounding color.

\subsection{Choice of focus color}

Focus color refers to the color of the local object that the operator looks at, such as the color of workpiece, product, mechanical operation part and operation center, etc.

According to the different nature of production, the focus color is also different. In the production process, products or workpieces have different colors, and they are processed under various backgrounds, which requires background color to have a prominent, eye-catching, easy to identify role.

Therefore, the color of the processed workpiece or product should be contrasted or complementary with the bottom color or background color. The most common metal products in the factory are cast iron, cast steel or aluminium products which background color is light brown. Similarly, when choosing the color of the operation parts, buttons and other recognition parts of the instrument and control board, we should also consider its impact on the safety and reliability of production. It is not easy to make mistakes by using strong and eye-catching colors. 
Focus color is also used in the movement part of machinery, operation center part and so on, which is the most important part of vision. It should be lighter than the surrounding color, so that the operator can unconsciously pay attention to this part, or can see it quickly and correctly.

Focus color is also used in the movement part of machinery, operation center part and so on, which is the most important part of vision. Its value should be higher than the surrounding color, so that the operator can unconsciously pay attention to this part, or can see it quickly and correctly.

\subsection{Choice of color for production equipment}

The color of production equipment is used for the color of various equipment in the workshop itself. It includes a variety of equipment (all kinds of machine tools, processing tools, etc.), movable cranes, workbenches, assembly lines and so on. Usually, non-stimulating, quiet color is used. But when the color of the workshop has been selected, you can choose the hue similar to or contrasted with it.

There are bright and gray colors. Generally, gray color feels heavier than bright one. The illusion of weight of these colors is caused by psychology. Lightness has the greatest influence on the weight of color. Gray looks heavy and bright feels light. Overweight will be depressing in people's psychology, so it is better to use bright color for heavy and huge machinery equipment. The base of machine tools is deep and heavy color to achieve stable visual sense, while the bed of machine tools mostly uses bright and soft tones. The movable crane should use light color to achieve flexible and light effect.

\subsection{Choice of surrounding color}

The surrounding colors mainly include the colors of the walls, floors and roofs of the workshop.

When choosing the interior color of workshop, besides the focus color and the color of production equipment, we should also pay attention to the surrounding color, give full play to the different characteristics of color, in order to improve, adjust and compensate for the shortcomings of the environment, and create a good and comfortable working environment.

Generally, warm tones should be used in the surrounding environment of cold processing workshop. If the color of production equipment is light blue, it is advisable to choose its weak contrast cream color or light orange yellow of warm tone as background color. This can not only clearly show their outlines, but also coordinate their colors. The calm cool tone is often used in the hot-working workshop to adjust the temperature sense of the workshop and improve the production environment.

However, neither cold processing workshop nor hot processing workshop should use too bright colors. Because it is not only easy to distract the operator's attention, but also easy to make the operator feel fatigued. Generally, the use of low-purity elegant and quiet color.

The relationship between illumination and color has been analyzed before. The influence of indoor illumination on color must be considered when choosing interior color in workshop. The wall is usually the background of the production equipment, which has a great influence on the light. Its color depends on the specific situation. Generally, light tones of low or medium purity, medium or slightly higher brightness are used. This kind of tone is suitable for harmonizing with doors and windows, production equipment and other objects. It is known from color science that low purity partial cold tone gives people a feeling of quiet, comfortable and cool, while low purity partial warm tone gives people a 
feeling of liveliness, cheerfulness and warmth. By fully utilizing some characteristics of color, the color design of workshop can be adapted to the requirements of work.

Through long-term research, Maurice Deribeli, a famous French chroma scientist, has put forward some useful suggestions on the color configuration of the walls and machine tools in the workshop. See the attached table, he believes that this kind of configuration is the most beneficial to human vision.

Table 1. Color Configuration of Workshop Internal Wall and Machine Tool[5].

\begin{tabular}{|c|c|c|c|c|}
\hline Wall color & Light yellow & Light yellow brown & Cream & Yellow Ochre \\
\hline $\begin{array}{c}\text { Machine Tool } \\
\text { Colors }\end{array}$ & Light Green & Sky Blue & Light Green & Light Blue \\
\hline
\end{tabular}

Because the common machine tools are concentrated in larger workshops, there are some noises and oil pollution in the environment, they usually use cold tones with low purity and moderate brightness. Precision machine tools and $\mathrm{CNC}$ machine tools or processing centers are placed in clean and quiet workshops, and they should adopt light tones or warm tones with high brightness and moderate purity, thus making the color of machine tools and environment an organic whole.

The ground is used to support a variety of production equipment, so the color can be used heavier. Medium gray or light colors are generally used to enhance stability.

Every workshop has a roof. The roof can choose white, milky white and other high brightness colors, but also can choose bright gray, light yellow or light blue and other colors with large reflection coefficient. Because the roof reflects light strongly, it is more important to see the environmental effect of color when choosing the color of the roof.

In the process of color design of workshop interior environment, attention should also be paid to the harmony and unification of the color used in the workshop interior with the overall color of the whole factory building and even the whole factory.

\section{Concluding remarks}

To sum up, the basic requirements of establishing a comfortable and safe production environment in the workshop are as follows:

1. To have a good light source, there should be enough illumination, and its distribution is uniform, no shadows, no dazzling, no strong contrast in the field of vision;

2. Deal with the relationship between color and illumination of production environment, and make them coordinate.

3. Deal with the relationship among focus color, production equipment color and surrounding environment color to achieve unity and harmony.

The interior environment color of workshop is composed of light, key color, various production equipment color and surrounding color. In order to create a color environment conducive to work and people's physical and mental health, these four factors can be considered organically for human purposes, so as to create a beautiful, comfortable, economical and reasonable production environment.

This research is supported by the "The research on education carrier and platform of engineering students innovative and entrepreneurship"(18- ZD-05) (2018-2020)

\section{Reference}

1. Yi-Feng Xin.Current Situation and Practice in Color Design of Building External Environment At home and abroad. http://www.civilcn.com/jianzhu/jzlw/jzjs/1491789836296615.html[2017-7-2] 
2. Lei Zhang,Bing-qing Xue, Yi Liu. A Preliminary Study on the Application of Color in the Art Design of Building External Environment.

http://www.chinaqking.com/yc/2019/1854883.html [2019-7-26]

3. Anonymous. Present Situation and Application of Color Design in Building External Environment. http://www.archcy.com/focus/colorful/be254c2c800f326a[2016-09-29]

4. Shuwen- Shi. Architectural environmental color design. China Construction Industry Press, 2014

5. Nenglin- Cheng. Product Modeling Design Manual, Machinery Industry Press, 2004 